\title{
Erratum to: Complications Associated with Botulinum Toxin Administration
}

\author{
Alexander Daoud and Martin Zaiac
}

\section{Erratum to:}

Chapter 13 in G. Fabbrocini et al. (eds.), Nonsurgical Lip and Eye Rejuvenation Techniques, DOI 10.1007/978-3-319-23270-6_13

Ivan Camacho is not a contributor to this chapter. Hence, the author's name and affiliation were removed. The updated authors names and affiliations should read as follows:

Alexander Daoud and Martin Zaiac

The online version of the updated chapter can be found at DOI 10.1007/978-3-319-23270-6_13

A. Daoud, BS

Greater Miami Skin and Laser Center,

Florida International University, Miami, FL, USA

M. Zaiac, MD ( $\varangle)$

Greater Miami Skin and Laser Center,

Florida International University,

Herbert Wertheim College of Medicine,

Miami Beach, FL, USA

e-mail: drmartyz@aol.com 